\title{
Ground States of Extended Hubbard Models in the Atomic Limit
}

\author{
U. Brandt and J. Stolze \\ Universität Dortmund, Institut für Physik, Dortmund, Federal Republic of Germany
}

Received September 26, 1985

\begin{abstract}
The possible ground states of an extended Hubbard model in the atomic limit, augmented by an additional nearest neighbour Ising-like interaction and an external magnetic field, are rigorously determined for arbitrary values of the coupling parameters and arbitrary chemical potential. The method used requires only simple convexity arguments and the examination of all possible configurations of small clusters of lattice sites, which may be done by computer. The results are valid for all lattices of $A B$ type (two interpenetrating sublattices). The types of order found are ferromagnetic, antiferromagnetic, and charge density wave. Perturbation theory suggests that for finite band width there may be a state showing both a charge density wave and ferromagnetic order.
\end{abstract}

\section{Introduction}

More than twenty years after its invention, the Hubbard model [1], introduced originally to describe electron correlation phenomena in narrow-band systems, is still being very actively investigated, as recent publications [2-4] show. The model, describing electrons in a simple tight-binding band interacting by an on-site Coulomb repulsion between electrons of opposite spin, was later augmented by a repulsion between electrons at nearest-neighbour sites; this model is usually called "extended Hubbard model" (see Eq. (1) below). The atomic limit (zero band width) was treated by Bari [5] who determined the ground states exactly for equal numbers of electrons and lattice sites and found a "Mott state" with one electron at each lattice site and a charge ordered state with vacant and doubly occupied sites in an alternating fashion. Bari also investigated a possible phase transition by a mean-field approximation. The same model was treated by Jędrzejewski [3] who proved the existence of a phase transition and derived a lower bound to the critical temperature by rigorous methods of statistical mechanics. Later the same author [4] treated this model on a square lattice for a general number of electrons. In addition to the ground state configurations mentioned above (and the trivial "completely filled" and "completely empty" states), he found a charge-ordered state with singly and doubly occupied sites in an alternating fashion and a similar one consisting of singly occupied and vacant sites. Furthermore, he proved the existence of several corresponding kinds of long range order for finite temperatures. The states discussed in [4] did not show any spin order, and Jędrzejewski raised the question how an additional magnetic interaction might change this situation. A model of this kind (with a nearest-neighbour Isinglike interaction between spins) was treated by Robaszkiewicz [6] in a mean-field approximation; Lorenz [7] gave a mean-field treatment of the finite band width case and found no coexistence of spin order (antiferromagnetism) and charge order.

In this paper, we determine the possible ground state configurations of an extended Hubbard model in the atomic limit, with an additional nearest-neighbour Ising-like interaction and an external magnetic field, for arbitrary interaction parameters and arbitrary chemical potential. We determine the regions of stability in parameter space for these various states and discuss the effects of a non-zero band width.

In Sect. II we introduce the model and transform it to an Ising system. In Sect. III we describe the method used to determine the possible ground state configurations. The method is based on simple energy 
and correlation arguments and is a variant of the one introduced in [8]; it permits us to find the ground states of the infinite lattice Hamiltonian by making a computer examine the finitely many configurations of a small cluster of lattice sites.

Section 4 contains the main results, namely the possible ground state configurations for all lattices of the $A B$-type (two interpenetrating sublattices) and their regions of stability, i.e. "ground state phase diagrams". It turns out that the introduction of the magnetic interaction does not create fundamentally new states but just removes some degeneracies in the states of [4]. There is no ground state in which a charge density wave (CDW) coexists with magnetic order, except at "phase boundaries". Some of the results of [6] obtained by a mean-field approximation are compared to our results. Finally, in Sect. V we use perturbation theory to lowest order in the band width to discuss how the ground state phase diagrams obtained in Sect. IV will be modified if the atomic limit is left. We conjecture that several disordered regions will show up in the phase diagram and that there may be a ferromagnetically ordered CDW phase, in contrast to the atomic limit.

\section{The Model}

We study a model defined by the Hamiltonian

$H=H_{H}+H_{M}$

where

$$
\begin{aligned}
H_{H}== & -t \sum_{\sigma=\uparrow, \downarrow} \sum_{\langle i, j\rangle}\left(c_{i \sigma}^{+} c_{j \sigma}+c_{j \sigma}^{+} c_{i \sigma}\right) \\
& +U \sum_{i} n_{i \uparrow} n_{i \downarrow}+V \sum_{\langle i, j\rangle} n_{i} n_{j}-\mu \sum_{i} n_{i}
\end{aligned}
$$

denotes the usual extended Hubbard model, and where

$$
\begin{aligned}
H_{M}:= & -J \sum_{\langle i, j\rangle}\left(n_{i \uparrow}-n_{i \downarrow}\right)\left(n_{j \uparrow}-n_{j \downarrow}\right) \\
& -B \sum_{i}\left(n_{i \uparrow}-n_{i \downarrow}\right)
\end{aligned}
$$

is an additional magnetic term. By $i=1, \ldots, N$ we denote the sites of a "simple cubic" lattice with dimension $d=2$ or $d=3$. On each site we have two pairs of fermion creation and annihilation operators $c_{i \sigma}^{+}, c_{i \sigma}(\sigma=\uparrow, \downarrow)$, furthermore

$n_{i \sigma}:=c_{i \sigma}^{+} c_{i \sigma}, \quad$ and $\quad n_{i}:=n_{i \uparrow}+n_{i \downarrow}$.

$\sum_{\langle i, j\rangle}$ denotes summation over all nearest-neighbour pairs, where each pair is counted once.
The first term in $H_{H}$ describes two degenerate tightbinding bands of width $4 d t$. We shall assume $t=0$ (atomic limit) for most of the remainder of this paper; only Sect. $V$ shall be devoted to the effects of nonvanishing band width. $U$ and $V$ characterize the intra-atomic and inter-atomic Coulomb energies, respectively. The chemical potential has been incorporated into the Hamiltonian because we wish to study systems of various electron densities between $n=0$ and $n=2$ electrons per site. The first term of $H_{M}$ describes an Ising-like interaction between spin densities at neighbouring sites, and $B$ is an external magnetic field. (A Heisenberg-like interaction, although physically more appropriate [9] is too complicated to be treated exactly.) Although $U$ and $V$ are positive by their physical nature, we wish to stress that our method is by no means restricted to this case. The ground state of the Hamiltonian (1) may be obtained for arbitrary values of the various parameters, as long as $t=0$.

For $t=0$ the model Hamiltonian (1) loses its quantum mechanical nature and there remain only the purely classical occupation numbers $n_{i \sigma}$. We are thus left with two interacting lattice gases, or equivalently, with two Ising models, one for each possible value of $\sigma$. For reasons of convenience we introduce two copies of the original lattice and place the "up" and "down" subsystems on different lattices. Of course there are interactions between the two lattices as well as within each lattice. The Hamiltonian (1) is transformed to a double Ising model by introducing two sorts of Ising spin variables

$\sigma_{i u}:=n_{i \uparrow}-1 / 2 ; \quad \sigma_{i d}:=n_{i \downarrow}-1 / 2$

corresponding to the "up" and "down" occupation numbers, respectively. The Hamiltonian thus may be written in the form

$$
\begin{aligned}
\frac{H}{N}= & J_{\|}+J_{\perp}+J_{s}-B_{u}-B_{d}-B_{u} m_{u}-B_{d} m_{d} \\
& -J_{\|} c_{\|}-J_{\perp} c_{\perp}-J_{s} c_{S} .
\end{aligned}
$$

The various quantities occurring in (3) are defined as follows:

$m_{u}:=\frac{1}{N} \sum_{i} 2 \sigma_{i u} \quad$ and $\quad m_{d}:=\frac{1}{N} \sum_{i} 2 \sigma_{i d}$

are the relative magnetizations of the $u$ and $d$ subsystems, respectively. The corresponding magnetic fields turn out to be

$B_{u}=\frac{1}{2}(\mu+B-z V-U / 2)$

and

$B_{d}=\frac{1}{2}(\mu-B-z V-U / 2)$, 
where $z$ is the coordination number of the lattice. The spin correlation between sites on the two Ising lattices corresponding to the same site of the original lattice is given by

$c_{\perp}:=\frac{1}{N} \sum_{i} 4 \sigma_{i u} \sigma_{i d}$,

the corresponding coupling constant is

$J_{\perp}=-U / 4$.

The nearest-neighbour correlation within the two Ising lattices is defined by

$c_{\|}:=\frac{4}{N z} \sum_{\langle i, j\rangle}\left(\sigma_{i u} \sigma_{j u}+\sigma_{i d} \sigma_{j d}\right)$,

accordingly

$J_{\|}=-z(V-J) / 4$.

The "diagonal" correlation between sites of the two Ising lattices corresponding to nearest-neighbour sites of the original lattice is

$c_{s}:=\frac{4}{N z} \sum_{\langle i, j\rangle}\left(\sigma_{i u} \sigma_{j d}+\sigma_{i d} \sigma_{j u}\right)$,

and finally

$J_{s}:=-z(V+J) / 4$.

The Ising model magnetizations and correlations are all normalized to the interval $[-1,1]$.

\section{The Method}

After the transformation described in the preceding section, the ground states of the model may be found by a method similar to the one developed in [8]. In order to fully understand the method, let us briefly recall the basic steps of the analysis in [8]. Every configuration of the Ising model corresponds to a point

$\mathbf{P}:=\left(m_{u}, m_{d}, c_{\|}, c_{\perp}, c_{s}\right)$

of $\mathbb{R}^{5}$. The set $S$ of all these points is convex in the thermodynamic limit, because the components of $N \mathbf{P}$ are extensive quantities.

In order to determine the shape of the set $S$ more precisely, linear inequalities of the form

$\mathbf{Q} \cdot \mathbf{P} \geqq \delta$

may be derived. These inequalities define a convex polyhedron (a simplex) which contains the desired set $S$.
Then one may find spin configurations corresponding to the extremal points (corners) of the simplex, thus showing that the set $S$ coincides with the simplex. As the energy (3) is a linear function of the components of $\mathbf{P}$, it assumes its extremal values at the corners of the simplex $S$ which are thus shown to correspond to possible ground state configurations of the system. We wish to stress that it is by no means certain that $S$ has to be a simplex with finitely many corners for an Ising system with finiterange interactions; at least we are not aware of a general proof of such a statement.

In [8] the necessary inequalities were proved analytically, a procedure which may become rather tiresome because it is not clear from the outset whether a given inequality will turn out to be relevant or not. To avoid this procedure, an algorithm was developed which generates a set of inequalities by inspection of all configurations of a cluster containing only a few spins. We shall now sketch this algorithm.

The procedure rests on the observation that the possible values of the correlations within a small cluster are less restricted than those of the infinite lattice. To make this more explicit, let us consider as an example three fixed sites $i, j, k$ of the original lattice, where $i$ and $k$ are nearest neighbours to $j$. As an analogue to the vector $\mathbf{P}$ introduced earlier, we define a vector $\mathbf{P}^{c}$ by its components

$$
\begin{aligned}
m_{u}^{c} & :=\frac{2}{3}\left(\sigma_{i u}+\sigma_{j u}+\sigma_{k u}\right) \\
m_{d}^{c} & :=\frac{2}{3}\left(\sigma_{i d}+\sigma_{j d}+\sigma_{k d}\right) \\
\mathcal{C}_{\mid:}^{c} & =2\left(\sigma_{i u} \sigma_{j u}+\sigma_{i d} \sigma_{j d}\right) \\
c_{\perp}^{c} & :=4 \sigma_{i u} \sigma_{i d} \\
c_{s}^{c} & :=2\left(\sigma_{j u} \sigma_{k d}+\sigma_{k u} \sigma_{j d}\right) .
\end{aligned}
$$

Obviously $\mathbf{P}^{c}$ can assume only finitely many points in $\mathbb{R}^{5}$; the convex hull of these points is a simplex $S^{c}$. We now show that $S^{c}$ contains the set $S$ defined above. To this end we consider an arbitrary (but fixed) configuration of the infinite lattice and calculate the vector $\mathbf{P}$ of this configuration in the following way. We translate (and rotate) the cluster $(i, j, k)$ through the whole lattice, determine $\mathbf{P}^{c}$ for each possible position (and orientation) of the cluster, and take the average to obtain $\mathbf{P}$. Thus every point $\mathbf{P}$ may be represented as a convex combination of points from $S^{c}$, which was to be shown. (On the other hand, $S^{c}$ may be considerably larger than $S$ : it is easy to construct spin configurations of the cluster $(i, j, k)$ yielding

$$
c_{\|}^{c}=c_{\perp}^{c}=1, \quad c_{s}^{c}=-1
$$


which is obviously impossible for the infinite lattice.) The inequalities defining the cluster simplex $S^{c}$ thus apply to the set $S$.

This way of deriving inequalities by computer offers a considerable amount of freedom which may be used to optimize the inequalities. Firstly, the size and shape of the cluster may be varied, and secondly, one may give different weights to the sites and bonds of the cluster. Part of these weights may even be negative, as long as their sum remains normalized.

As an output we obtain a list of inequalities and a list of points, both describing the simplex $S^{c}$. We may then try to construct a configuration of the infinite lattice the $\mathbf{P}$-vector of which is equal to a corner of $S^{c}$, thereby determining a possible ground state of the system. If necessary, we may repeat the procedure with another type of cluster to gain more inequalities.

\section{Results}

The methods introduced in the preceding section may be easily applied to the model given by Eqs. (38). It suffices to consider the simplest conceivable cluster, namely two nearest-neighbour sites $i$ and $j$, defining

$$
\begin{aligned}
m_{u}^{c} & :=\sigma_{i u}+\sigma_{j u}, \quad m_{d}^{c}:=\sigma_{i d}+\sigma_{j d} \\
c_{\|}^{c} & :=2\left(\sigma_{i u} \sigma_{j u}+\sigma_{i d} \sigma_{j d}\right) \\
c_{\perp}^{c} & :=2\left(\sigma_{i u} \sigma_{i d}+\sigma_{j u} \sigma_{j d}\right) \\
c_{s}^{c} & :=2\left(\sigma_{i u} \sigma_{j d}+\sigma_{i d} \sigma_{j u}\right) .
\end{aligned}
$$

With very little numerical effort one then obtains the simplex $S^{c}$, the corners of which are listed in Table 1 . The corresponding configurations of the infinite lattice will be discussed below.

Table 1. The corners of the simplex $S^{c}$

\begin{tabular}{lrrrrll}
\hline Corner No. & $m_{u}$ & $m_{d}$ & $c_{\|}$ & $c_{\perp}$ & $c_{s}$ & Configuration \\
\hline 1 & 1 & 1 & 1 & 1 & 1 & $\mathrm{i})$ \\
2 & -1 & -1 & 1 & 1 & 1 & $\left.\mathrm{i}^{\prime}\right)$ \\
3 & 1 & 0 & 0 & 0 & 0 & $\mathrm{ii})$ \\
4 & 0 & 1 & 0 & 0 & 0 & $\mathrm{ii})$ \\
5 & 0 & -1 & 0 & 0 & 0 & $\left.\mathrm{ii}^{\prime}\right)$ \\
6 & -1 & 0 & 0 & 0 & 0 & $\left.\mathrm{ii}^{\prime}\right)$ \\
7 & 1 & -1 & 1 & -1 & -1 & iii $)$ \\
8 & -1 & 1 & 1 & -1 & -1 & iiia) \\
9 & 0 & 0 & -1 & -1 & 1 & iii b) \\
10 & 0 & 0 & -1 & 1 & -1 & iv) \\
\hline
\end{tabular}

The inequalities defining the simplex $S^{c}$ may all be written in the form

$$
\mathbf{P} \cdot \mathbf{Q} \geqq-1
$$

with the $\mathbf{Q}$ vectors given in Table 2 .

In the last column we have listed those corners from Table 1 which fulfill the inequality $\left(9^{\prime}\right)$ as an equality.

Table 2. The inequalities defining the faces of the simplex $S^{c}$ (sce main text for details)

\begin{tabular}{lrrrrrrrrrrr}
\hline $\begin{array}{l}\text { Inequality } \\
\text { no. }\end{array}$ & \multicolumn{1}{c}{ Components of $\mathbf{Q}$} \\
\hline 1 & 0 & 0 & -1 & 1 & -1 & 1 & 2 & 7 & 8 & 9 & \\
2 & 0 & 0 & -1 & -1 & 1 & 1 & 2 & 7 & 8 & 10 & \\
3 & 1 & -1 & 0 & 0 & -1 & 1 & 2 & 4 & 6 & 8 & 9 \\
4 & -1 & 1 & 0 & 0 & -1 & 1 & 2 & 3 & 5 & 7 & 9 \\
5 & -1 & 1 & 1 & -1 & -1 & 1 & 2 & 3 & 5 & 9 & 10 \\
6 & 1 & -1 & 1 & -1 & -1 & 1 & 2 & 4 & 6 & 9 & 10 \\
7 & -1 & -1 & 0 & 1 & 0 & 1 & 3 & 4 & 7 & 8 & 9 \\
8 & 1 & 1 & 0 & 1 & 0 & 2 & 5 & 6 & 7 & 8 & 9 \\
9 & -1 & -1 & 1 & 0 & 0 & 1 & 3 & 4 & 9 & 10 & \\
10 & 1 & 1 & 1 & 0 & 0 & 2 & 5 & 6 & 9 & 10 & \\
11 & -1 & -1 & 1 & 1 & 1 & 3 & 4 & 7 & 8 & 9 & 10 \\
12 & 1 & 1 & 1 & 1 & 1 & 5 & 6 & 7 & 8 & 9 & 10 \\
13 & 1 & -1 & 1 & 0 & 0 & 4 & 6 & 8 & 9 & 10 & \\
14 & -1 & 1 & 1 & 0 & 0 & 3 & 5 & 7 & 9 & 10 & \\
15 & 1 & -1 & 0 & -1 & 0 & 1 & 2 & 4 & 6 & 8 & 10 \\
16 & -1 & 1 & 0 & -1 & 0 & 1 & 2 & 3 & 5 & 7 & 10 \\
17 & -1 & -1 & 0 & 0 & 1 & 1 & 3 & 4 & 7 & 8 & 10 \\
18 & 1 & 1 & 0 & 0 & 1 & 2 & 5 & 6 & 7 & 8 & 10 \\
\hline
\end{tabular}

The fact that the simplex $S$ may be determined by using a two-site cluster has a very important consequence. It means that the ground states (as given by the macroscopic quantities making up the vector P) are not only the same for the square and simple cubic lattices, but are also identical for all lattices which may be divided into two sublattices $A$ and $B$ with every $A$-site being surrounded by $B$ sites and vice versa ( $A B$ lattices), for example honeycomb ( $d$ $=2$ ) or bcc $(d=3)$ lattices. (One may consider even more general networks, provided that the coordination number is the same for all vertices of the network, and that all closed paths consist of an even number of elementary steps.)

We now consider the corners listed in Table 1. Obviously, to every corner with non-vanishing $m_{u}$ or $m_{d}$ there exists a "mirror image" (marked by a prime) with reversed signs of both $m_{u}$ and $m_{d}$, due to the spin reversal symmetry of the Ising model. Another symmetry operation of this point set is the interchange of $m_{u}$ and $m_{d}$, corresponding to spin reversal in the original extended Hubbard model. The infinite lattice configurations corresponding to the corners from Table 1 are all determined uniquely, up to symmetry operations. This is trivial for configurations of types i) and iiia), which are completely determined by the values of $m_{u}$ and $m_{d}$. For configura- 
tions iii b) and iv), $c_{\|}=-1$ implies that every bond within each of the $u$ and $d$ subsystems connects oppositely oriented spins; $c_{\perp}= \pm 1$ characterizes the two possible ways in which the two generalized checkerboard patterns generated by $c_{\|}=-1$ may be "stacked". The uniqueness of the configurations of type ii) may be derived from those inequalities which are fulfilled as equalities by these corners. (Obviously, the corners of the simplex $S$ are defined by the fact that sufficiently many inequalities become equalities.) Considering corner no. 3 of Table 1 , we see that $m_{u}=1$ fixes the $u$ subsystem. Furthermore this corner fulfills the inequality

$\left|m_{u}+m_{d}\right| \leqq 1+c_{\|}+c_{\perp}+c_{s}$

(nos. 11/12 of Table 2) as an equality. We now prove (10) analytically to see what this inequality implies for the spin configuration. To this end we consider a lattice site $i$ and one of its nearest neighbours, $j$, say to the right of $i$. We then have

$m_{u}+m_{d}=\frac{1}{N} \sum_{i}\left(\sigma_{i u}+\sigma_{j u}+\sigma_{i d}+\sigma_{j d}\right)$.

Using the triangle inequality and the inequality

$|n| \leqq n^{2}$

valid for integer $n$, we obtain

$\left|m_{u}+m_{d}\right| \leqq \frac{1}{N} \sum_{i}\left(\sigma_{i u}+\sigma_{j u}+\sigma_{i d}+\sigma_{j d}\right)^{2}$

from which (10) follows by collecting terms of the square expression (and repeating the argument for different possible directions of the bond between $i$ and $j$ ). For (10) to become an equality, both the triangle inequality and (12) have to become equalities. This means that the terms of the sum (11) are only allowed to assume the values zero and \pm 1 and that they must have the same sign throughout the lattice. In combination with the given values of $m_{u}$ and $m_{d}$ this fixes the configuration corresponding to corner no. 3 of Table 1.

The resulting five different types of configuration for the Hubbard model may be characterized as follows:

i) is a homogeneously charged unmagnetic state; all sites of the Hubbard lattice are doubly occupied ( $n$ $=2$ ).

ii) is a spin-polarized charge-density wave (CDW) state, consisting of doubly and singly occupied sites in an alternating fashion $(n=3 / 2)$; the spins of the singly occupied sites are aligned.

iiia) is a homogeneously charged ferromagnetic state, consisting of singly occupied sites $(n=1)$ with all spins parallel. iii $b$ ) is a homogeneously charged antiferromagnetic state, consisting of singly occupied sites $(n=1)$ with adjacent spins antiparallel.

iv) is an unmagnetic CDW state consisting of doubly occupied and vacant sites in an alternating fashion.

We thus see that there is a spin-polarized CDW state, but no antiferromagnetic state with non-trivial charge order. (Of course there may be coexistence of antiferromagnetic and CDW states for special combinations of the interaction parameters; see below.) The spin polarization of the $n=\frac{3}{2}$ CDW state, however, will break down as the external magnetic field is turned off (see below).

"Ground state phase diagrams" may be easily constructed by determining which of the states listed in Table 1 yields the lowest energy at a given point in coupling parameter space. The vectors $\mathbf{Q}$ given in Table 2 correspond to points of coexistence of several phases: at the point

$\left(B_{u}, B_{d}, J_{\|}, J_{\perp}, J_{s}\right)=-|\varepsilon| \mathbf{Q}$

at least five of the corners given in Table 1 have the energy per site

$\frac{H}{N}=J_{\|}+J_{\perp}+J_{s}-B_{u}-B_{d}-|\varepsilon|$

due to (3) and $\left(9^{\prime}\right)$, and none of the remaining corners has a lower energy. Thus Table 2 may be used to find the most interesting regions in the five-dimensional space of coupling parameters of the model. In the last column of Table 2 we have listed the configurations which coexist at points related to a given $\mathbf{Q}$ vector by (14).

Let us now discuss the extended Hubbard model without external magnetic field. This amounts to fixing $B_{u}=B_{d}$ in the Ising picture, and so no new ground state configurations will occur. Some pairs of ground state configurations from Table 1 , however, will now be degenerate, namely 3 and 4,5 and 6,7 and 8 . The degeneracy between corners 7 and 8 is indeed twofold, because, $c_{\|}=1$ and $c_{\perp}=-1$ imply ferromagnetic order in the Hubbard model, whereas the direction of the magnetization is not fixed. The situation is totally different for the configurations of type ii) (i.e. 3-6): without external field the spins of the singly occupied sites are not fixed; the charge order however remains, as the inequality (10) and its proof are still valid. This results in a total degeneracy of $2\left(\frac{N}{2}+1\right)$ for each of the two surviving states ii)

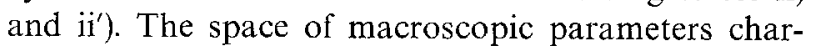
acterizing the Ising model states now is four-dimen- 
Table 3. The inequalities for the extended Hubbard model without external magnetic field (see main text for details)

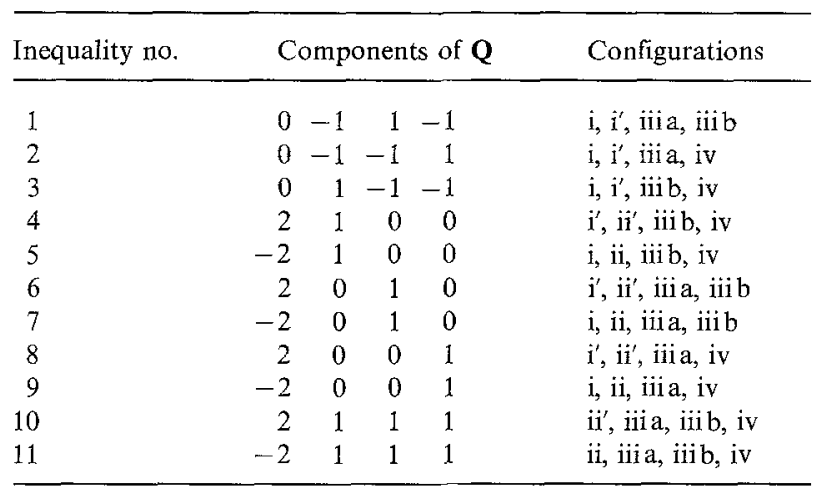

sional, with the coordinates

$m:=\left(m_{u}+m_{d}\right) / 2$,

and $c_{\|}, c_{\perp}, c_{s}$ defined as before. The four-dimensional $\mathbf{Q}$-vectors listed in Table 3 again characterize the faces of the simplex as well as the coexistence points in ground state phase diagrams. In Fig. 1 we have tried to visualize the structure of the ground-state phase diagram for the zero-field model. Note that one of the four coupling parameters of the model may be fixed, thus defining energy units. We have set $B \equiv+1$ (in the Ising picture), which is equivalent to

$\mu-\mu_{0}=+1$

in the Hubbard picture. Here

$\mu_{0}:=z V+U / 2$

denotes the value of chemical potential for which the Hamiltonian (1) shows particle-hole symmetry. The phase diagram for negative values of $\mu-\mu_{0}$ may be obtained from the one given in Fig. 1 by interchanging particles and holes (and using $\mu_{0}-\mu$ as energy unit). We have used $U /\left(\mu-\mu_{0}\right)$ and $z(V$ $\pm J) /\left(\mu-\mu_{0}\right)$ as parameters for the ground state phase diagram.

In Fig. 1, each of these parameters lies in the range between -1 and +3 . The resulting cube contains all phases which are stable for $\mu>\mu_{0}$. The stability region of the $n=3 / 2$ CDW state (configuration ii) is a regular tetrahedron with the corners

$$
\begin{aligned}
& \frac{1}{\mu-\mu_{0}}(z(V-J), U, z(V+J)) \\
& \quad=(2,0,0),(0,2,0),(0,0,2),(2,2,2) .
\end{aligned}
$$

On each of the faces of the tetrahedron the type-iiphase coexists with one of the four remaining types

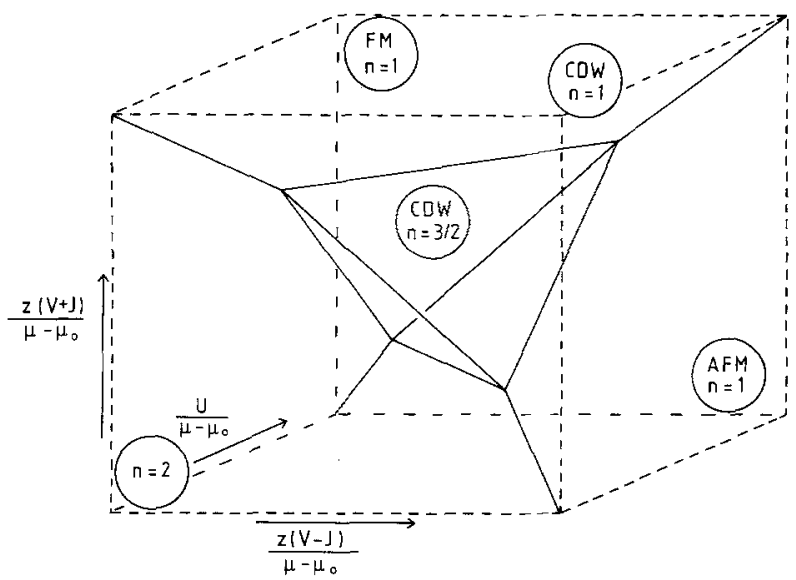

Fig. 1. Ground state phase diagram of the extended Hubbard model. Each of the variables ranges from -1 to +3

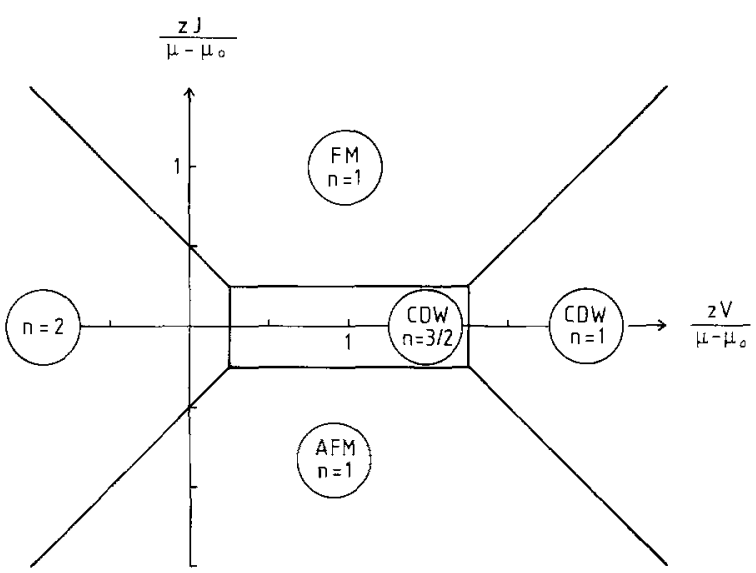

Fig. 2. Ground state phase diagram for $U /\left(\mu-\mu_{0}\right)=3 / 2, \mu>\mu_{0}$

of configuration. The six coexistence planes between any two of these four phases touch one of the edges of the tetrahedron and cut one of the faces of the cube along its diagonal. (The face diagonals of the cube have been omitted in Fig. 1 for the sake of clarity.) The six coexistence planes meet at four "triple lines" along body diagonals of the cube. The various phases in Fig. 1 have been characterized by their particle densities $n$ (average number of electrons per site) and the types of charge or spin order. (AFM and FM denote antiferromagnetic and magnetic order, respectively.) Figure 2 shows a typical section of Fig. 1 (at $U /\left(\mu-\mu_{0}\right)=3 / 2$ ), however, with rotated axes in order to represent the Hubbard model parameters more directly.

The phase diagram derived in [4] for $J=0$ cannot be obtained directly as a two-dimensional section of Fig. 1, because in [4], $V$ was chosen as energy unit, but of course our results reduce to those of [4] for $J$ $=0$. Comparing our results to those of [4], we see that the magnetic interaction $J$ changes the highly 


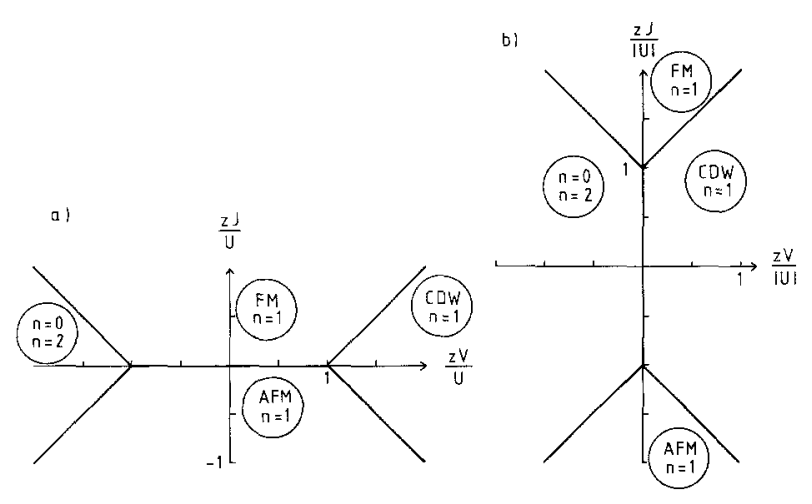

Fig. 3a and b. Ground state phase diagram for $\mu=\mu_{0} ;$ a: $U>0$, b: $U<0$

degenerate spin-disordered $n=1$ phase into two magnetically ordered phases. The spin-disordered $n$ $=3 / 2 \mathrm{CDW}$ phase, however, is not affected by $J$, because in this phase the spin density vanishes identically on one of the two sublattices. Only a magnetic field can align the spins of the singly occupied sites, leading to the two spin-polarized CDW states ( 3 and 4 of Table 1) mentioned earlier. However, it would be misleading to call these states ferromagnetic, as their polarization is enforced by an external field. We thus see that for vanishing magnetic field coexistence of magnetic order and charge density waves can only occur at boundaries in the phase diagram, i.e. on certain planes in Fig. 1. (See however Sect. $V$ for the effects of a finite band width.)

It is of some interest to compare our results to the $T$ $=0$ parts of the results of Robaszkiewicz [6] who studied the present model in a mean-field approximation. Let us first study the system at the point of particle-hole symmetry, $\mu=\mu_{0}$. Figures $3 \mathrm{a}$, b show the ground state phase diagrams for this case, with positive and negative $U$, respectively.

For positive values of $V$ these diagrams agree with the results of [6]; the region of coexistence between the $n=0$ and $n=2$ phases, however, is not discussed there. These two coexisting phases will separate in space, as the nearest neighbour attraction $V<0$ favours the clustering of doubly occupied sites. Only at $V=0$ and $U \leqq 0$ a disordered state (a random mixture of doubly occupied and vacant sites) may occur for $|z J|<|U|$.

The foregoing argument may be generalized to discuss the type of coexistence (spatially separated phases or mixture) for other points of degeneracy between different phases. We have seen earlier that every possible ground state of the system is charac-
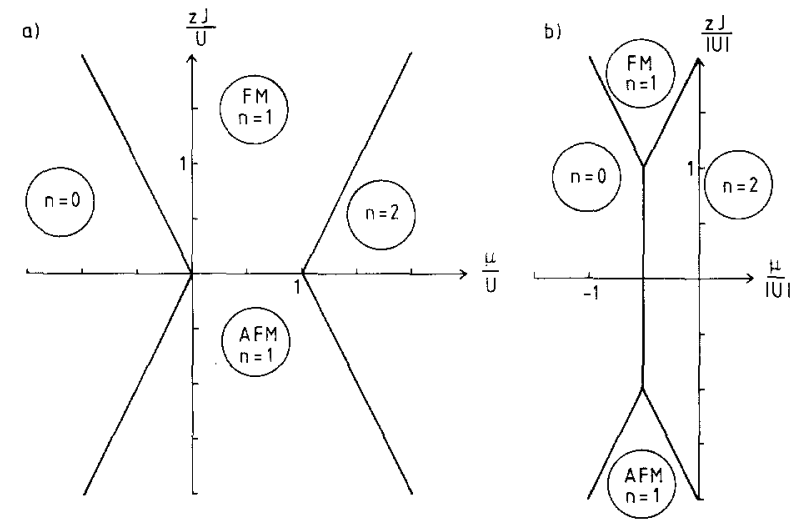

Fig. 4.a and b. Ground state phase diagram for $V=0 ; \mathbf{a}: U>0$, b: $U<0$

terized by some conditions which every nearestneighbour pair of sites has to fulfill. Thus a point of coexistence between different structures may be understood as a point of coexistence between different sorts of nearest-neighbour pair configurations. Now, if it is possible to construct an interface between two coexisting structures without introducing forbidden pair configurations, then the two phases will easily mix, otherwise they will not. By this argument we see immediately that the ferromagnetic and antiferromagnetic states will mix at coexistence, forming a paramagnetic state, whereas the two degenerate ferromagnetic phases will not mix but rather form polarized domains. Similarly we see that the two magnetically ordered states will not mix with any of the remaining states of Fig. 3 (which contain no singly-occupied sites).

Let us now turn to the case $V=0$. The corresponding phase diagrams are displayed in Figs. $4 \mathrm{a}, \mathrm{b}$. The possible ground states are those with $n=0, n=2$, and the ferromagnetic and antiferromagnetic $n=1$ states. A paramagnetic state exists for $U>0, J=0$ and $0 \leqq \mu \leqq U$, a charge-disordered state (mixture of doubly occupied and vacant sites) exists for $\mu=U / 2$, $|z J| \leqq-U(U<0)$. At the remaining phase boundaries, spatial phase separation occurs.

From the phase boundaries one may easily derive the results for the chemical potential which are summarized in the following table:

\begin{tabular}{l|l|l|}
\multicolumn{1}{c}{$U<0$} & \multicolumn{1}{l}{$U>0$} \\
\cline { 2 - 3 } $0<n<1$ & $\mu=\min (-z|J| / 2, U / 2)$ & $\mu=-z|J| / 2$ \\
\cline { 2 - 3 } $1<n<2$ & $\mu=\max (U / 2, U+z|J| / 2)$ & $\mu=U+z|J| / 2$ \\
\cline { 2 - 3 } & &
\end{tabular}

Thus neither the chemical potential nor the phase boundaries depend on $n$ (apart from the distinction 

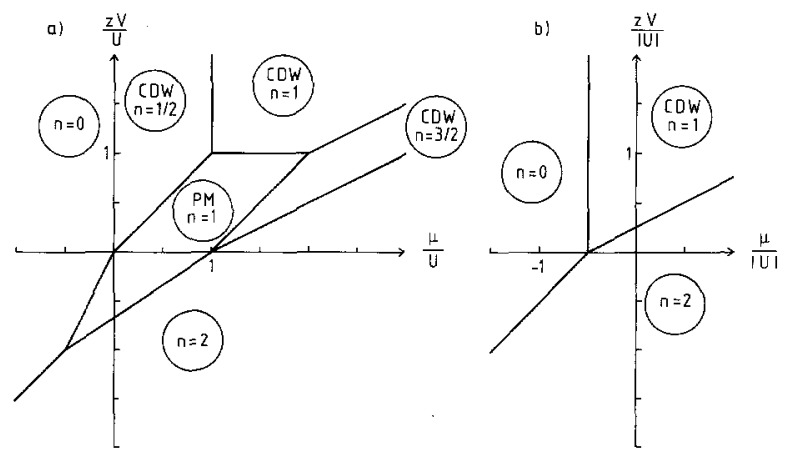

Fig. 5a and b. Ground state phase diagram for $J=0$; a: $U>0$, b: $U<0$

between $n>1$ and $n<1$ ), in contrast to the meanfield results in [6]. One may speculate whether this failure of the mean-field approximation may be traced back to the assumption of translational invariance (of the average electron occupation etc.) within each sublattice which is obviously not fulfilled for phase boundaries where non-miscible phases coexist.

We now consider the case $J=0$ (treated also in [4]), with the phase diagram shown in Figs. $5 \mathrm{a}, \mathrm{b}$. The paramagnetic (PM) state occurring in Fig. 5 a results from the degeneracy between the two magnetically ordered $n=1$ states. Disordered states may exist at the boundaries of the $n=1 / 2$ and $n=3 / 2$ CDW regions in Fig. $5 \mathrm{a}$ and at the boundaries of the $n=1$ $\mathrm{CDW}$ region in Fig. 5 b. Again, the mean-field approximation [6] for this case yields $n$-dependent phase boundaries, whereas the exact phase boundaries obviously are "piecewise $n$-independent".

\section{Perturbation Theory for Non-Zero Band Width}

We conclude the discussion of our results by some qualitative considerations concerning the influence of a non-vanishing band width $t \neq 0$ within lowestorder perturbation theory. We need not consider the states $n=2, n=0$ and $n=1$, ferromagnetic, because these states obviously are eigenstates of the Hamiltonian (with $t$-independent energies) also for finite band width. The regions of stability of these states, however, may be indirectly affected by a finite band width, because other states may become lower in energy as the band width increases.

To first order in $t$, the remaining "pure" ground states are also unaffected, because application of $c_{i \sigma}^{+} c_{j \sigma}$ (if possible) to any of these states changes electron occupation at two mutually adjacent sites and thus leads to a state lacking the strictly periodic electron occupation characteristic of the ground states. The situation is different, however, with parameter combinations allowing for coexistence of two or more different ground state configurations. In these cases of high degeneracy the ground state contains interfaces between regions of different configuration. The hopping term may have non-zero matrix elements between ground states differing only in electron occupation of two nearest-neighbour sites at an interface, and thus there may be a first order correction to the ground state energy. One may expect a breakdown of the unperturbed ground state especially with those parameter combinations for which the coexisting phases are completely miscible, whereas in the immiscible cases the perturbation may be considered a negligible surface effect.

We now discuss the second-order correction to the ground state energy for the $n=1 \mathrm{CDW}$ state, the $n$ $=3 / 2 \mathrm{CDW}$ state and the $n=1$ antiferromagnetic state. As these states are degenerate, we have to use degenerate perturbation theory and thus have to diagonalize the matrix with elements

$\sum_{\nu} \frac{\left\langle 0\left|H_{1}\right| v\right\rangle\left\langle v\left|H_{1}\right| 0^{\prime}\right\rangle}{E_{0}-E_{v}}$.

Here $|0\rangle$ and $\left|0^{\prime}\right\rangle$ are two degenerate ground states, $\{|v\rangle\}$ is a basis in the space of (unperturbed) excited states, and the $E$ 's are unperturbed energies. It is easy to see that the off-diagonal elements of (17) vanish for all three states mentioned above and we are left with the usual second order expression for the diagonal elements always yielding a lowering of the ground state energy. The states $|v\rangle$ contributing to the second order energy correction (per site) $\varepsilon_{2}$ are easily constructed, yielding

$\varepsilon_{2}=z t^{2} /[(2 z-1) J+V-U]$

for the antiferromagnetic state, and

$\varepsilon_{2}=z t^{2} /[U-(2 z-1) V+J]$

for the $n=1 \mathrm{CDW}$ state. The energy corrections (18) and (19) are equal if the two states coexist, i.e. at $U$ $=J+V$ and thus this phase boundary is not changed by a small finite band width.

For the $n=3 / 2$ CDW phase the situation is slightly more difficult. This phase is highly degenerate because the spins of the $N / 2$ singly occupied sites are arbitrary. The ground state energy obviously is independent of $J$, as the doubly occupied sites possess no spin. The intermediate states $|v\rangle$ in (17) are now constructed by transferring one electron of spin $\sigma$ from a doubly occupied site to a nearest neighbour site occupied by one electron of spin $-\sigma$. This process creates $z-1$ nearest-neighbour pairs with non- 
vanishing magnetic interaction. The corresponding contribution to $E_{v}$ obviously depends on the local spin configuration, that is, on the choice of $|0\rangle$ (out of $2^{\left(\frac{N}{2}+1\right)}$ possible states) and of $|v\rangle$ (out of $N z / 2$ possible states for given $|0\rangle)$. We thus may write for the $n=3 / 2 \mathrm{CDW}$ state

$N \varepsilon_{2}=\sum_{v=1}^{N z / 2} t^{2} /\left[-(z-1) V-g_{0 v} J\right]$

where $g_{0 v}$ may assume the values $\pm(z-1), \pm(z-3)$ etc. As the second order ground state energy correction is not the same for all spin configurations of the singly occupied sublattice, we may ask whether the finite band width favours spin order. From Fig. 1 we recall that the projection of the stability region of the $n=3 / 2$ CDW phase onto the $\frac{z}{\mu-\mu_{0}}(V, J)$ plane is a square with corners $(0,0),(1,1),(2,0)$, and $(-1,1)$; thus $V$ is non-negative throughout this region, and $|J| \leqq V$. For the state showing complete ferromagnetic order in the singly occupied sublattice all energy denominators in (20) assume the value $-(z-1)(V-J)$, yielding the lowest possible energy for $J>0$. We thus see that a non-vanishing band width may lead to ferromagnetic order in a CDW phase. The second-order energy correction diverges for $J \rightarrow V$. This is not surprising, since a look at Fig. 1 reveals that at $J=V$ the $n=3 / 2 \mathrm{CDW}$ (ferromagnetic) phase gives way to the $n=1$ ferromagnetic and $n=2$ phases.

For $J<0$ it is obviously unfavourable to have a spin-polarized sublattice of singly occupied sites. Let us consider for a given unperturbed ground state $|0\rangle$ those $z$ intermediate states $|v\rangle$ which are created by transferring an electron from a given doubly occupied site to a nearest neighbour site; if $g$ of these sites have spin $\sigma$ and $(z-g)$ sites have spin $-\sigma$, then the total contribution of the corresponding states to the ground state energy correction (20) is given by

$$
\begin{aligned}
& N \Delta \varepsilon_{2}(g):=t^{2} g /[(1-z) V-J(z+1-2 g)] \\
& +t^{2}(z-g) /[(1-z) V-J(z+1-2(z-g))] .
\end{aligned}
$$

Inspection of this expression shows that its minimum is situated either at $g=1$ or at $g=z / 2$ (for even $z$ ), or $g=(z \pm 1) / 2$ (for odd $z$ ). The minimum occurs at $g=1$ for

$J / V<-(z-1) /(z+1) ;$

thus for sufficiently strong antiferromagnetic coupling (near the borderline to the $n=2$ and $n=1$ antiferromagnetic phases) and for sufficiently small coordination number we obtain an "almost ferromagnetic" phase with one reversed spin among the $z$ nearest neighbours of every doubly occupied site. For weaker antiferromagnetic coupling a "locally balanced" paramagnetic phase results. The $n=1 / 2$ CDW phase may be discussed in an entirely analogous way, with identical phase boundaries in the $(J, V)$-plane.

Summarizing the above discussion we may draw some tentative conclusions about the modifications which will result in the phase diagram of Fig. 1 due to a finite band width. The $n=3 / 2$ CDW phase will be modified as discussed above, leading to a coexistence of magnetic order and charge order. The faces of the tetrahedrical stability region of this phase (where in the atomic limit coexistence of miscible phases occurs) will broaden to form layers containing various kinds of disordered phases. The same will happen at the boundaries between the ferromagnetic and antiferromagnetic phases and between the $n=2$ and $n=1 \mathrm{CDW}$ phases. At the boundaries between the ferromagnetic and $n=1 \mathrm{CDW}$ phases and between the antiferromagnetic and $n=2$ phases the exact eigenstates of the $t \neq 0$ Hamiltonian (i.e. the ferromagnetic and $n=2$ phases) will be pushed back, as their energies do not decrease with increasing band width. The boundaries between the ferromagnetic and $n=2$ and between the antiferromagnetic and $n=1$ CDW states will not change at all.

\section{References}

1. Hubbard, J.: Proc, R. Soc. London, Ser. A 276, 238 (1963); 281, 401 (1964); for numerous applications see, for example: Electron correlation and magnetism in narrow-band systems. In: Springer Series in Solid-State Sciences. Moriya, T. (ed.). Vol. 29. Berlin, Heidelberg, New York: Springer 1981

2. Hirsch, J.E.: Phys. Rev. Lett. 51, 1900 (1983); Phys. Rev. B31, 4403 (1985)

3. Jędrzejewski, J.: Z. Phys. B - Condensed Matter 48, 219 (1982)

4. Jedrzejewski, J.: Z. Phys. B - Condensed Matter 59, 325 (1985)

5. Bari, R.A.: Phys. Rev. B 3, 2662 (1971)

6. Robaszkiewicz, S.: Acta Phys. Polon. A 55, 453 (1979)

7. Lorenz, B.: Phys. Status Solidi (b) 106, K 17 (1981)

8. Brandt, U.: Z. Phys. B - Condensed Matter 53, 283 (1983)

9. Compare, for example Mattis, D.C.: The theory of magnetism I. In: Springer Series in Solid State Sciences. Vol. 17, Chap. 6. Berlin, Heidelberg, New York: Springer Verlag 1981

U. Brandt

J. Stolze

Institut für Physik

Universität Dortmund

Postfach 500500

D-4600 Dortmund 50

Federal Republic of Germany 